\title{
La motivación de logro mejora el rendimiento académico
}

\author{
José Miguel García Ramírez. Universidad de Granada \\ Recepción: 23 de diciembre de 2015 | Revisión: 26 de diciembre de 2015 | Aceptado: 26 de diciembre de 2015 \\ Correspondencia: miguelgr@ugr.es \\ Citar: Garcia-Ramirez, JM. (2016). La motivación de logro mejora el rendimiento académico. ReiDoCrea, 5, 1-8.
}

\begin{abstract}
Resumen: A través de los proyectos de innovación docente las universidades mejoran y consolidan la calidad de la educación que ofrecen, tomando la creatividad y visibilidad como claves de la motivación por competencias y logro. Método: La investigación se realiza durante el curso académico 2013-14, a través del proyecto de innovación docente "ReiDoCrea" de la Universidad de Granada. Los participantes ( $N=62, \mathrm{M}=22.7, \mathrm{SD}=6.6$ ), estudiantes de la Facultad de Ciencias del Trabajo, se adscribieron voluntariamente al Grupo Experimental $(n=30)$ o al Grupo Control ( $n=32)$. El grupo experimental participó en el proyecto, mientras que el grupo control no participó; los instrumentos utilizados fueron dos pruebas objetivas (pre y post) del programa de evaluación académica. Resultados: El análisis estadístico muestra que existen diferencias significativas entre los resultados obtenidos por el Grupo Experimental $(M=83.6, S E=1.259, t(60)=3.748$, $p<.05, d=.95 r=.43)$ y el Grupo Control $(M=77.25$, $S E=1.14)$. Conclusiones: En la innovación docente, la motivación por competencias y de logro es clave para desarrollar el pensamiento creativo y mejorar el rendimiento académico.
\end{abstract}

Palabras clave: Innovación Pedagógica | Motivación

Achievement Motivation Improves Academic Performance

\begin{abstract}
Through teaching innovation projects universities improve and consolidate the quality of education they offer, taking creativity and visibility as the key to competence and achievement motivation. Method: The research study was conducted during the academic year 2013-14, through the teaching innovation project "ReiDoCrea" of the University of Granada. The participants $(\mathrm{N}=62, \mathrm{M}=22.7, \mathrm{SD}=6.6)$, students of the Faculty of Labour, voluntarily joined the Experimental Group $(n=30)$ or the Control Group $(n=32)$. The experimental group participated in the project, while the Control Group did not participate; the instruments used were two objective tests (pre and post), part of the academic program evaluation. Results: The statistical analysis shows that there are significant differences between the results obtained by the Experimental Group $(\mathrm{M}=83.6, \mathrm{SE}=1.259, t(60)=3.748, p<.05, d=.95 r=.43)$ and the Control Group (M=77.25, $\mathrm{SE}=1.14)$. Conclusions: In teaching innovation, competence and achievement motivation is key to developing creative thinking and improving academic achievement.
\end{abstract}

Keywords: Teaching Method Innovations | Motivation

\section{Introducción}

La innovación se asocia con la creatividad la cual es clave para que el sistema educativo afronte los cambios en la sociedad e incorpore una cultura de calidad y excelencia docente en la Educación Superior (UNESCO, 1998). Así que las universidades tienen la responsabilidad social de asegurar la transmisión del conocimiento contextualizado y actualizado (Garcia-Ramirez, 2011; Bozu y Canto 2009), para lo que es necesario promocionar el pensamiento divergente como proceso cognitivo complejo necesario para la resolución de problemas de cualquier índole. Teniendo en cuenta que este proceso cognitivo se desarrolla a través de los factores de fluidez, flexibilidad, originalidad y elaboración, junto al desarrollo de la imaginación como facultad mental en la que se fomentan la capacidades de fantasía, intuición y asociación (Sternberg, 1999; Guilford, 1950). Por lo tanto, el pensamiento divergente conlleva el desarrollo de habilidades y actitudes relacionadas con la docencia, dando lugar a un profesorado de actitudes flexibles, dominio de los contenidos y competencias didácticas (Fiorini y Garcia-Ramirez, 2013; Torre, 2009); un profesorado que es capaz de generar intervenciones innovadoras, cuya producción creativa puede ser evaluada a través de las categorías de: a) novedad 
que incluye los criterios de originalidad, germinalidad y transformacionalidad; b) resolución que incluye los criterios de lógica, adecuación, eficacia, utilidad y valor; c) elaboraciónsíntesis que incluye los criterios la expresividad, atracción, organización y parsimonia (Kaufman, \& Sternberg, 2010; Amabile, 1983).

Los proyectos de innovación docente ayudan a conseguir y asegurar una enseñanza renovada e interdisciplinar en la Sociedad del Conocimiento, que junto a las Tecnologías de la Información y la Comunicación -TIC-, los entornos virtuales y las redes sociales se posibilita el acceso a las bases de datos para la construcción y la divulgación del conocimiento (Molina, 2012; Salinas, 2004). Aún así, el profesorado es quien tiene la capacidad de transmitir el conocimiento y crear las inquietudes necesarias para que el aprendizaje sea intencional; por lo que el uso de las TIC, los entornos virtuales y las redes sociales son complementos de gran ayuda en la evolución educativa y que concretamente en ámbito universitario incrementan la productividad (Garcia-Ramirez, 2012; Menchen, 2009).

Algunas investigaciones realizadas para conocer la actitud de los docentes hacia la inclusión de las nuevas tecnologías en la docencia han mostrado que su actitud y percepción influyen en uso de estas en la innovación docente. Las TIC, los entornos virtuales de aprendizaje personal y el uso de herramientas didácticas ayudan a diseñar actividades educativas dentro de los parámetros del modelo constructivista, mejorando la innovación docente y los factores que motivan, interesan y refuerzan el aprendizaje en el alumnado (Diaz-Marin, Vazquez-Martinez \& McMullin, 2014; Espuny, Gonzalez, Lleixa y Gisbert, 2011). De todas maneras la intencionalidad en el proceso de enseñanzaaprendizaje es la que facilita la adquisición, construcción y divulgación del conocimiento. Por lo tanto, la interacción entre profesorado y alumnado, dentro de un contexto de interdependencia positiva y de motivación intrínseca, conlleva la adquisición y desarrollo de competencias y de logro (Garcia-Ramirez, 2014; Weiner, 1990; McClelland, 1984).

En la Universidad de Granada, a partir del curso académico 2011-12, se empezó a implementar el proyecto de innovación docente "ReiDoCrea", de corte constructivista, con la misión de desarrollar la motivación de logro. En este proyecto el profesorado participante anima a su alumnado a publicar los trabajos de investigación que realizan durante el curso académico. Esto supone una mejora global en el proceso de enseñanzaaprendizaje, ya que implica de forma participativa y creativa a ambas partes del proceso educativo, en la que el profesorado participante asume la responsabilidad de orientar y guiar a sus estudiantes durante todo el proceso, para que estos adquieran los conocimientos necesarios y competencias propias de su área del conocimiento. Finalmente la publicación de los trabajos supone transparencia docente y reconocimiento público, lo que mejora la percepción de eficiencia y satisfacción. Para implementar este proyecto, previamente, se tenía que incluir en la guías docentes de las asignaturas que imparten el profesorado participante, siendo la participación voluntaria y sin ningún incremento adicional a la calificación final; o sea los trabajos del alumnado participante son evaluados con los mismos criterios que los realizados por el alumnado no participantes; la motivación por competencias forma parte del currículo académico y el profesorado tiene la obligación de sus estudiantes adquieran y desarrollen las competencias necesarias para realizar estudios de investigación, ni puede ni debe beneficiar a nadie fuera de lo establecido curricularmente, ya que eso supondría trato de favor; la única diferencia es que los/as participante se benefician de la motivación de logro. En este proyecto se usa la metodología de investigación-acción, porque esta permite resolver los problemas que surgen durante el desarrollo, asegurando la 
finalización (Elliott, 1993). También se usan las TIC, redes sociales y entornos de aprendizaje personal como medios para acceder a las bases de datos y dar visibilidad y transparencia al conocimiento adquirido. (Garcia-Ramirez, 2012, 2013, 2014, 2015)

Este estudio de investigación exploratorio tiene como hipótesis que la motivación de logro mejora el rendimiento académico.

\section{Método}

\section{Participantes}

Sesenta y dos estudiantes (49 mujeres y 13 hombres) del Grado de Relaciones Laborales de la Facultad de Ciencias de Ciencias de Trabajo de la Universidad de Granada, con edades comprendidas entre 20 y 52 años $(N=62, M=22.69 ; S D=6.59)$. Los datos se muestran a continuación en la tabla 1.

\begin{tabular}{|cccccc|}
\hline \multicolumn{6}{|c|}{ Tabla 1. Estadísticos descriptivos } \\
\hline & N & Mínimo & Máximo & Media & Desv. típ. \\
\hline Edad & 62 & 20 & 52 & 22,69 & 6,590 \\
\hline
\end{tabular}

\section{Instrumentos}

Los instrumentos usados fueron dos pruebas objetivas, tipo test, de contenido teórico cuyos ítems fueron obtenidos de los 6 capítulos que componen la asignatura obligatoria de "Desarrollo de Personas y Grupos en las Organizaciones" del Grado de Relaciones Laborales. La primera prueba, P1, consistía en contestar 36 ítems, de cuatro alternativas, obtenidos de los tres primeros capítulos y la segunda prueba, P2, consistía en contestar 36 ítems, de cuatro alternativas, obtenidos de los tres últimos capítulos.

\section{Procedimiento}

Este estudio se desarrolla durante el segundo semestre del curso académico 2013-14, en un grupo de tercer año del Grado de Relaciones Laborales de la Universidad de Granada. Los/as participantes tenían que realizar las dos pruebas objetivas anteriormente descritas en el apartado de instrumentos y también tenía que realizar un trabajo de investigación empírica relacionado con los tres últimos capítulos teóricos, todo estaba recogido en la guía docente de la asignatura.

La primera prueba, $\mathrm{P} 1$, se tenía que hacer en la última semana de marzo; después los estudiantes tenían que realizar un trabajo de investigación que tendrían que exponer durante la última semana de mayo; finalmente tenían que realizar la segunda prueba, P2, durante la primera semana de junio.

Durante la primera semana de clase el alumnado fue informado de todo el proceso, expilándoles que sus trabajos de investigación podían adscribirse, con carácter voluntario, al proyecto de Innovación Docente "ReiDoCrea", esto les supondría transcribir su estudio en forma de artículo y después enviarlo a la Revista electrónica de investigación Docencia-Creativa para su evaluación y posible publicación; para ello, tendría que cumplir con las normas de publicación de esa revista. Esta participación en el proyecto de 
innovación ni los eximia de presentar su trabajo en clase durante la última semana de mayo ni les supondría un incremento adicional en la calificación del trabajo; esto se hacía para evitar que la muestra estuviese sesgada por un posible beneficio en la calificación final y sobre todo para asegurar que realmente los/as participantes actuases influenciados/as por la motivación de logro.

Los/as participantes $(\mathrm{N}=62)$, después de realizar la primera prueba $(\mathrm{P} 1)$, se constituyeron en grupos de trabajo y voluntariamente decidieron si se adscribían al proyecto ReiDoCrea. Los/as participantes que se adscribieron al proyecto constituyeron el Grupo Experimental "GE" $(n=30)$ y el resto de los/as participantes constituyeron el Grupo Control "GC" $(n=32)$.

Los/as participantes finalizaron y expusieron sus trabajos en la última semana de mayo; posteriormente realizaron la segunda prueba objetiva (P2).

\section{Análisis de datos}

Los datos obtenidos de los resultado de las dos pruebas objetivas, P1 y P2, se analizaron con el programa estadístico SPSS 20: primero se realizó la prueba de normalidad de Shapiro-Wilk, después se realizó la prueba de Levene para la igualdad de varianzas y finalmente la prueba T de Student para muestras independientes. Luego se calculó $d$ de Cohen o tamaño de efecto para el mismo grupo experimental antes y después de la implementación del proyecto de innovación docente y posteriormente se calculó la $r$ o potencia estadística para conocer el tamaño del efecto.

\section{Resultados}

Antes de proceder a realizar el análisis estadístico, los resultados obtenidos se muestran en tres diagramas radiales en la figura 1.

El primer diagrama muestra los resultados obtenidos de ambas pruebas en ambos grupos, comparándolos. Se visualiza que los datos obtenidos de los resultados obtenidos de la segunda prueba respecto a los datos obtenidos de los resultados de la primera prueba son mayores en el grupo experimental. Sin embargo en el grupo control se da el efecto contrario los datos obtenidos de los resultados de la primera prueba son ligeramente mayores que los datos obtenidos de la segunda prueba.

Después se muestran dos diagramas en los que se visualizan los datos obtenidos de los resultados de cada prueba, P1 y P2, para cada grupo por separado, pudiéndose apreciar la tendencia de los resultados de la evaluación con y sin participación en el proyecto de innovación docente.

Los diagramas radiales sólo muestran una apreciación visual de que existen diferencias entre los datos obtenidos de los resultados de ambas pruebas para ambos grupos. Esa apreciación anticipa el resultado positivo del análisis estadístico de que las evidencias, que visualmente se aprecian, pueden ser significativas y por tanto se cumpliría la hipótesis de partida de este estudio de investigación. 
Diagrama radial de los resultaos obtenidos de ambas pruebas, P1 y P2, para ambos grupos, GC y GE.

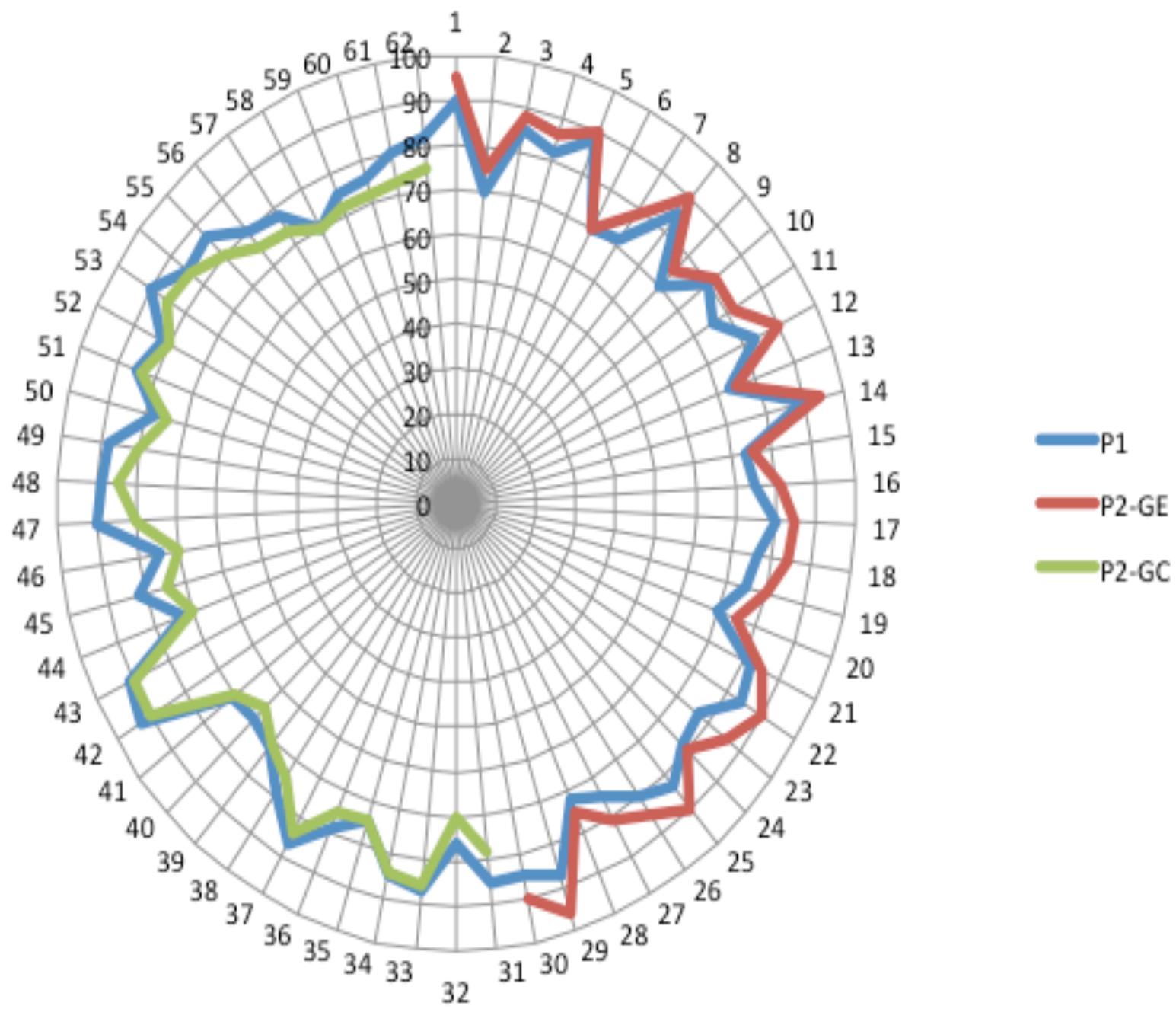

Diagrama radial de los resultados obtenidos para ambas pruebas, P1 y P2, en el Grupo Control, GC.

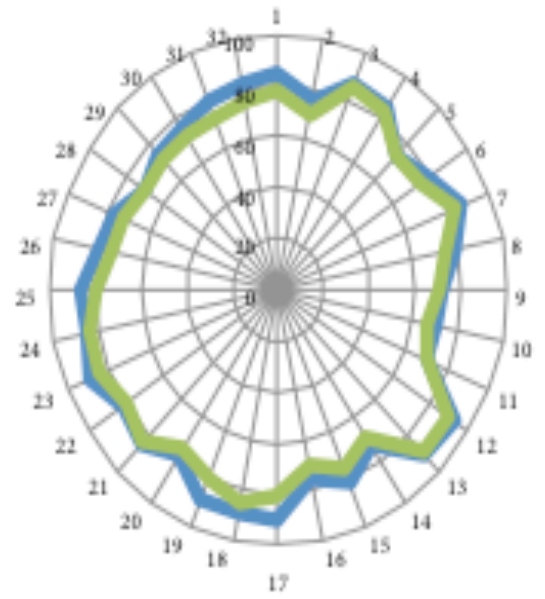

Diagrama radial de los resultados obtenidos para ambas pruebas, P1 y P2, en el Grupo Experimental, GE.

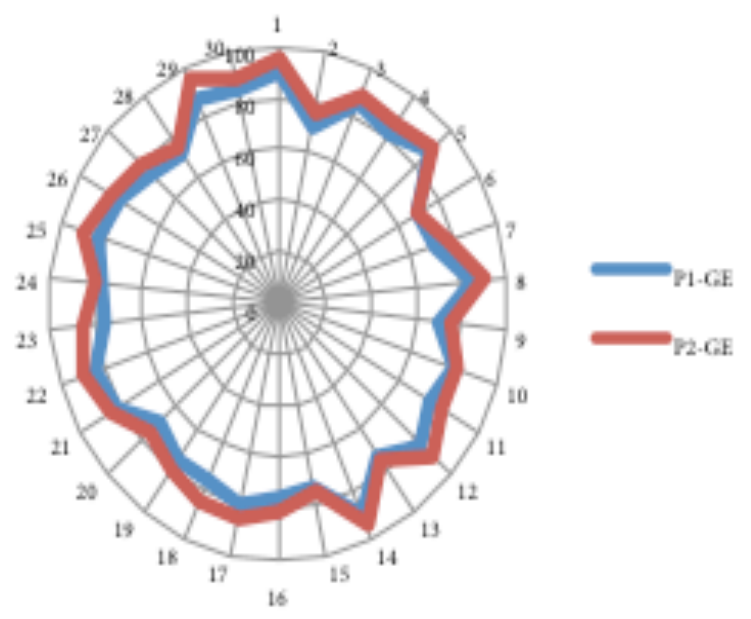


A continuación se procede a analizar los datos con el programa estadístico SPSS 20. Primero se realiza la prueba de normalidad de Shapiro-Wilk (tabla 2), para comprobar que la significancia es mayor de 0.05 ( $p>.05)$ para ambos grupos (P1-GC: $p=.316, \mathrm{P} 1-\mathrm{GE}$ : $p=.118$, P2-GC: $p=.065$, P2-GE: $p=.248$ ); por lo tanto, se confirma que los datos provienen de una distribución normal.

\begin{tabular}{|llccc|}
\hline \multicolumn{4}{|c|}{ Tabla 2. Pruebas de normalidad } \\
\hline \multirow{2}{*}{ GRUPO } & \multicolumn{3}{c|}{ Shapiro-Wilk } \\
\cline { 2 - 5 } & & Estadístico & gl & Sig. \\
\hline P1 & Control &, 962 & 32 &, 316 \\
\cline { 2 - 5 } & Experimental &, 944 & 30 &, 118 \\
\hline P2 & Control &, 938 & 32 &, 065 \\
\cline { 2 - 5 } & Experimental &, 956 & 30 &, 248 \\
\hline *. Este es un límite inferior de la significación verdadera. \\
a. Corrección de la significación de Lilliefors \\
\hline
\end{tabular}

Después, se procede a comparar estadísticamente las medias de los datos obtenidos de la ambas pruebas, a través de la prueba T para muestras independientes. Los resultados indican en la prueba de Levene para la igualdad de varianzas, se asumen varianzas iguales para ambas pruebas ( $\mathrm{P} 1: \mathrm{F}=.1, p=.752 ; \mathrm{P} 2: \mathrm{F}=.7, p=.792)$.

La prueba T para la P1 indica que no existen diferencias significativas entre los resultados obtenidos de participantes del Grupo Control (M=81.03, $S E=1.175)$ y el Grupo Experimental $(\mathrm{M}=78.87, \mathrm{SE}=1.143)$. Por consiguiente antes de implementar el proyecto de Innovación Docente ReiDoCrea no existían diferencias significativas entre ambos grupos.

Sin embargo la prueba T para la P2 indica que sí existen diferencias significativas entre los resultados obtenidos de los participantes del Grupo Control $(M=77.25, S E=1.139)$ y el Grupo Experimental $(\mathrm{M}=83.6, \mathrm{SE}=1.259, t(60)=3.748, p<.00, d=.95, r=.43)$ con una potencia ligeramente grande que explica un tamaño del efecto del $25 \%$ aprox. de la varianza total. Por lo tanto, se puede afirmar, después de realizar el análisis estadístico, que la motivación de logro influye positivamente en la calificación de la segunda prueba, cumpliéndose la hipótesis de partida de este estudio. A continuación se muestra el análisis estadístico con más detalle en las tablas 3 y 4 .

\begin{tabular}{|cccccc|}
\hline \multicolumn{5}{c|}{ Tabla 3. Estadísticos de grupo } \\
\hline & GRUPO & $\mathrm{N}$ & Media & Desviación típ. & Error típ. de la media \\
\hline P1 & Control & 32 & 81,03 & 6,645 & 1,175 \\
\cline { 2 - 6 } & Experimental & 30 & 78,87 & 6,263 & 1,143 \\
\hline P2 & Control & 32 & 77,25 & 6,446 & 1,139 \\
\cline { 2 - 6 } & Experimental & 30 & 83,60 & 6,896 & 1,259 \\
\hline
\end{tabular}

\begin{tabular}{|c|c|c|c|c|c|c|c|c|c|c|}
\hline \multicolumn{11}{|c|}{ Tabla 4. Prueba de muestras independientes } \\
\hline & & \multicolumn{2}{|c|}{$\begin{array}{c}\text { Prueba de Levene para la } \\
\text { igualdad de varianzas }\end{array}$} & \multicolumn{7}{|c|}{ Prueba T para la igualdad de medias } \\
\hline & & \multirow[t]{2}{*}{$\mathrm{F}$} & \multirow[t]{2}{*}{ Sig. } & \multirow[t]{2}{*}{$\mathrm{t}$} & \multirow[t]{2}{*}{$\mathrm{gl}$} & \multirow[t]{2}{*}{$\begin{array}{c}\text { Sig. } \\
\text { (bilateral) }\end{array}$} & \multirow[t]{2}{*}{$\begin{array}{l}\text { Diferencia } \\
\text { de medias }\end{array}$} & \multirow[t]{2}{*}{$\begin{array}{c}\text { Error típ. } \\
\text { de la } \\
\text { diferencia }\end{array}$} & \multicolumn{2}{|c|}{$\begin{array}{c}95 \% \text { Intervalo de } \\
\text { confianza para la } \\
\text { diferencia }\end{array}$} \\
\hline & & & & & & & & & Inferior & Superior \\
\hline & $\begin{array}{l}\text { Se han asumido } \\
\text { varianzas iguales }\end{array}$ & 100 & ,752 & 1,318 & 60 & 193 & 2,165 & 1,643 & $-1,121$ & 5,450 \\
\hline & $\begin{array}{l}\text { Se han asumido } \\
\text { varianzas iquales }\end{array}$ & ,070 & ,792 & $-3,748$ & 60 & ,000 & $-6,350$ & 1,694 & $-9,739$ & $-2,961$ \\
\hline
\end{tabular}




\section{Discusión}

El proyecto de innovación docente ha sido significativamente eficiente y positivo en los/as participantes del Grupo Experimental. El análisis estadístico de los datos obtenidos en este estudio de investigación demuestra que la motivación de logro influye positivamente en el rendimiento académico. Por lo tanto, se puede recomendar que en Educación Superior se apueste por los proyecto de innovación docente que implique motivación de logro en sus participantes, como el caso del proyecto de innovación docente ReiDoCrea.

La innovación docente es clave para que la Educación sea de Calidad y Excelencia, pero tiene que ser visible, puesto que la visibilidad del trabajo y del esfuerzo profesorado y alumnado en el proceso complejo de enseñanza-aprendizaje, genera satisfacción y sentido de eficiencia, que son dos elementos necesarios para desarrollar la motivación por competencias y de logro.

Por supuesto, es necesario que el profesorado forme, guie y oriente a su alumnado en la realización de sus trabajos, estudios e investigación; pero también es necesario crear y desarrollar interacción entre profesorado y alumnado, porque esa interacción es la que está relacionada significativamente con la satisfacción y el sentido de la eficiencia. La empatía y simpatía entre profesorado y alumnado, ayuda a construir colaborativamente el conocimiento. De esta manera, también, se potencia el desarrollo del pensamiento creativo, teniendo como base el contexto y los conocimientos previamente adquiridos. (Garcia-Ramirez, 2012; Flaherty, 2010)

Por lo tanto, las universidades, a través de los proyectos de innovación docente y el uso de las nuevas tecnologías, pueden mejorar la calidad de la docencia que ofertan; también en es uso la metodología de investigación-acción en la educación ayuda a abordar y resolver los problemas que van surgiendo durante la docencia de manera inmediata.

La educación, como proceso de enseñanza-aprendizaje, necesita crear programas, proyectos de innovación docente que incrementen la satisfacción y la motivación tanto en el profesorado como en el alumnado; es necesario crear, desarrollar e implementar instrumentos que mejoren el rendimiento académico como es el caso del proyecto de Innovación Docente ReiDoCrea que a través del uso de las nuevas tecnología creó una revista electrónica de investigación, para dar visibilidad y transparencia al esfuerzo y rendimiento académico. La revista no más que es un instrumento que ha servido y sirve para construir colaborativamente el conocimiento y potenciar la motivación por competencias y de logro personal tanto en el alumnado como en el profesorado participante.

Concluyendo, en la innovación docente, la motivación por competencias y de logro es clave para desarrollar el pensamiento creativo y mejorar el rendimiento académico.

\section{Referencias}

Amabile, TM. (1983). The social psychology of creativity: A componential conceptualization. Journal of Personality and Social Psychology, 45(2), 357-376.

Bozu, Z. y Canto, P. (2009). El profesorado universitario en la sociedad del conocimiento: competencias profesionales docentes. Revista de Formación e Innovación Educativa Universitaria, 2(2), 87-97. 
Diaz-Marin, V., Vazquez-Martinez, A., \& McMullin, K. (2014). First steps towards a university social network on personal learning environments. The International Review of Research in Open and Distance Learning, 15(3), 93-119.

Elliott, J. (1993). El cambio educativo desde la investigación-acción. Ediciones Morata.

Espuny, C., Gonzalez, J., Lleixa, M. y Gisbert, M. (2011). Actitudes y expectativas del uso educativo de las redes sociales en los alumnos universitarios. Revista de Universidad y Sociedad del Conocimiento (8)1, 171-185.

Fiorini, M. y García-Ramírez, JM. (2013). Cap. 5: Técnicas de grupo y creatividad aplicadas en el ámbito universitario,117147. En M.D. Villena Martínez y A. Muñoz García (2013). Recursos para la tutoría en el aula universitaria. Granada: Editorial Universidad de Granada.

Flaherty, J. (2010). Coaching: Evoking Excellence in Others. Routledge.

Garcia-Ramirez, JM. (2011). Una reconsideración de la excelencia visible en la educación superior: la escucha empática. Andaluciaeduca, 66, 84.

Garcia-Ramirez, JM. (2012). Docencia-Creativa: una estrategia de motivación a través de la difusión del conocimiento. ReiDoCrea, 1, 1-3.

Garcia-Ramirez, JM. (2012). La comunicación, clave de excelencia visible en la Educación Superior. Journal for Educators, Teachers and Trainers, 3, 25-36.

Garcia-Ramirez, JM. (2012). Las Tecnologías de la Información y la Comunicación, TIC, en la educación universitaria. Andaluciaeduca, 76, 77.

Garcia-Ramirez, JM. (2013). ReiDoCrea un proyecto de construcción y divulgación del conocimiento. ReiDoCrea, 2, 1-10.

Garcia-Ramirez, JM. (2014). ReiDoCrea, un proyecto de investigación-acción. ReiDoCrea, 3, 1-3.

Garcia-Ramirez, JM. (2014). Selección de indicadores para la evaluación de la excelencia docente en la Universidad de Trent (Canadá). Granada: Universidad de Granada.

Garcia-Ramirez, JM. (2015). ReiDoCrea, un proyecto de innovación en la transparencia docente. ReiDoCrea, 4, 1-5.

Guilford, J.P. (1950). Creativity. American Psychology, 14, 469-479

Kaufman, J.C., \& Sternberg, R.J. (Eds.). (2010). The Cambridge Handbook of Creativity. Cambridge University Press.

McClelland, DC. (1984). Human Motivation. Oakland, NJ: Scott Foresman.

Menchen, F. (2009). La creatividad y las nuevas tecnologías en las organizaciones modernas. Madrid: Díaz de Santos.

Molina, AM. (2012). Las TIC en la educación superior como vía de formación y desarrollo competencial en la sociedad del conocimiento. ReiDoCrea, 1, 106-114.

Salinas, J. (2004). Innovación docente y uso de las TIC en la enseñanza universitaria. Revista Universidad y Sociedad del Conocimiento, 1(1), 1-16.

Sternberg, RJ. (Ed.). (1999). Handbook of Creativity. Cambridge University Press.

Torre, S. (2009). La universidad que queremos. Estrategias creativas en el aula universitaria. Revista Digital Universitaria, 10(12), 1-17.

UNESCO (1998). Declaración Mundial sobre la Educación Superior en el S XXI: Visión y Acción. Conferencia Mundial sobre Educación Superior. UNESCO.

Weiner, B. (1990). History of Motivational Research in Education. Journal of Educational Psychology, 82, 616-622. 